\title{
Impact of Anal Fissure on Neuroticism, Extraversion, Openness to Experience, Agreeableness, and Conscientiousness: A Case-Control Study
}

\author{
Paloma Luri-Prieto $^{\mathrm{a}}$ Asunción Candela-Gomis ${ }^{\mathrm{a}}$ Antonio Palazón-Bru ${ }^{\mathrm{b}}$ \\ Felipe Navarro-Cremades ${ }^{b}$ Vicente Francisco Gil-Guillén ${ }^{b}$ \\ Antonio Fernando Compañ-Rosique ${ }^{c}$

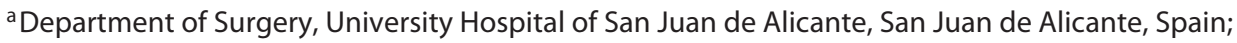 \\ ${ }^{b}$ Department of Clinical Medicine, Miguel Hernández University, San Juan de Alicante, Spain; ' $D e p a r t m e n t ~ o f$ \\ Pathology and Surgery, Miguel Hernández University, San Juan de Alicante, Spain
}

\begin{abstract}
Keywords
Fissure-in-ano · Neuroticism · Extraversion, psychological · Openness · Agreeableness · Conscientiousness · Personality inventory Personality tests
\end{abstract}

$0.91,95 \% \mathrm{Cl} 0.83-1.00, p=0.049$, AUC 0.69). Conclusions: The cases mainly presented higher levels of neuroticism and lower levels of openness to experience and conscientiousness. Further studies are needed to corroborate our results.

(c) 2020 S. Karger AG, Basel

\section{Introduction}

An anal fissure is a linear ulcer that extends from the pectinate line to the margin of the anus affecting the anoderm lining the lower half of the internal anal sphincter. Most of these fissures are primary or idiopathic and must be differentiated from fissures secondary to other conditions. The clinical characteristic is pain during defecation, which frequently persists afterwards [1]. Although anal fissures are common in surgical practice (representing between 6 and $15 \%$ of consultations and $10 \%$ of interventions), their etiopathogenesis remains controversial, as the sympathetic and enteric nervous systems are involved [2]. An acute anal fissure tends to heal in 1-2 weeks with proper hygiene and dietary measures. A chronic fissure is defined as a fissure that does not heal after 6 weeks of con- 
servative treatment and requires pharmacological and/or surgical treatment $[3,4]$.

A personality trait is defined as a stable and enduring individual characteristic that differentiates people by their tendencies to show consistent thought patterns, emotions, and behaviors [5]. The 5 personality domains (neuroticism, extraversion, openness, agreeableness, and conscientiousness) are the main focus of the Big Five model. Neuroticism can be defined as a relatively stable tendency to view the world and one's life negatively, to respond with negative emotions and high levels of arousal to experiences of threat, frustration, or loss [6]. Extraversion is associated with diverse interests and social dimensions. Openness to experience involves the need for variety, newness, and change. Agreeableness is associated with attitudes of forgiveness and belief in cooperation. Conscientiousness is related to having an organized support network and technical expertise. Of the 5 factors, neuroticism is the trait related to psychopathology [7]. In our clinical practice we have detected certain psychological characteristics that are common among patients with anal fissure. Our aim was to further explore the personality of this group of patients in order to respond to our clinical concern and compare our results with those in the literature. Consequently, to corroborate our hypothesis, we carried out a case-control study to determine certain common (prevalent) psychological traits, such as neuroticism.

\section{Materials and Methods}

\section{Study Population, Design, and Participants}

Outpatients diagnosed with acute or chronic idiopathic anal fissure in the healthcare area of San Juan University Hospital (Alicante, Spain) were included. This healthcare area covers a total of 218,299 inhabitants (figures from June 30, 2018), providing free and universal coverage for the diagnosis, monitoring, and treatment of this condition.

These patients receive hygiene recommendations and dietary treatment as the first step in their treatment. After the fissure has been diagnosed as chronic, pharmacological treatment is initiated with topical nitrates and/or calcium antagonist ointments in order to achieve chemical sphincterotomy. Failure of this second therapeutic step leads to an indication for surgical intervention with a lateral internal sphincterotomy.

This case-control study was carried out in the healthcare area of San Juan (Alicante, Spain) between January 2016 and February 2017. Cases were defined as any patient diagnosed with acute or chronic idiopathic anal fissure during the study period who wished to participate in the study and provided written informed consent. A fissure was considered to be chronic when symptoms were present for $>6$ weeks or there were signs of chronicity, that is, at least one of the following findings was present: indurated margins, visible internal anal sphincter fibers, sentinel skin tags, or hypertrophied anal papillae. Exclusion criteria were fissures secondary to other diseases such as Crohn's disease, HIV infection, tuberculosis, syphilis, herpes, carcinoma, leukemia, agranulocytosis, and hidden abscesses. In addition, patients with functional alterations (incontinence or constipation), rectocele or prolapse, or those who had undergone previous surgical treatment for an anorectal disorder or birth trauma were excluded. A clinical diagnosis of severe psychiatric disorder, and specifically of depressive disorder or anxiety disorder, was also an exclusion criterion.

The controls were selected from patients who had a surgery consultation for a condition other than anal fissure and from hospital personnel (doctors, nurses, and administrative staff). In other words, the controls came from the population from which the cases arose, which minimized possible selection bias. As with the cases, all control group participants gave written informed consent to participate in the study. The same exclusion criteria were applied for the controls, including chronic pain and/or history of surgery.

\section{Variables and Measurements}

The instrument used to describe the personality traits was the NEO Five-Factor Inventory (NEO-FFI), a short version of the NEO personality inventory-revised (NEO PI-R) [6]. The NEO-FFI is a psychometric instrument that evaluates the 5 dimensions of personality without considering the thirty facets measured in the complete test. It is composed of 60 items scored according to a 5-point Likert-type scale, ranging from total disagreement to total agreement and corresponding to the following 5 basic personality factors: neuroticism, extraversion, openness, agreeableness, and conscientiousness. The higher the score for each of these factors, the more the subject is considered to possess this personality trait. The NEO-FFI has been validated in Spanish, with good validity and reliability indicators [8].

The questionnaire was completed by both cases and controls. The cases filled in the questionnaire at the time of the diagnosis of the fissure and the controls at the time of recruitment. A single investigator administered all the questionnaires and resolved any doubts that arose. The sex and age of the participants were also recorded.

\section{Sample Size Calculation and Statistical Methods}

A pilot study was carried out with 14 cases and 14 controls to estimate the mean neuroticism score on the NEO-FFI questionnaire, as we found no similar studies that quantified these values. In this pilot study, the mean neuroticism score was $23.07 \pm 8.54$ in the cases and $14.71 \pm 6.96$ in the controls. With these data, assuming a type I error of $5 \%$, a power of $99 \%$, and an allocation ratio of one control per case, it was found that at least 32 cases and 32 controls were needed.

The variables were described using absolute and relative frequencies (qualitative) and means with standard deviation (quantitative). The means of the different measurements of the questionnaire between cases and controls were compared using the Student $t$ test. The odds ratios (OR) adjusted for sex and age were obtained for each of the measurements, determining how well the model fit the data (goodness-of-fit using the Hosmer-Lemeshow test) and the discriminatory capacity of the model (area under the receiver operating characteristic curve, AUC). All analyses were performed with a significance of $5 \%$ and for each relevant parameter its associated confidence interval was calculated. The calculations were implemented in IBM SPSS Statistics 25 and $\mathrm{R}$ 3.3.3.

\section{Results}

A total of 67 people participated in the study, of whom $35(52.2 \%)$ were cases and the rest were controls (Table 1). Of the total sample, $53.7 \%(n=36)$ were men 
Table 1. Descriptive and bivariate analysis of the study sample

\begin{tabular}{lcccr}
\hline Variables & Total $(n=67)$ & Cases $(n=35 ; 52.2 \%)$ & Controls $(n=32 ; 47.8 \%)$ & $p$ \\
\hline Sex & & & & 0.117 \\
$\quad$ Male & $36(53.7)$ & $22(62.9)$ & $14(43.8)$ & \\
$\quad$ Female & $31(46.3)$ & $13(37.1)$ & $51.4 \pm 11.5$ & 0.618 \\
Age, years & $52.2 \pm 12.1$ & $52.9 \pm 12.7$ & $13.4 \pm 6.1$ & $<0.001$ \\
Neuroticism & $17.1 \pm 7.9$ & $20.6 \pm 7.9$ & $28.9 \pm 7.1$ & 0.066 \\
Extraversion & $27.3 \pm 6.7$ & $25.9 \pm 6.1$ & $29.2 \pm 6.7$ & 0.005 \\
Openness to experience & $26.6 \pm 7.4$ & $24.3 \pm 7.3$ & $32.1 \pm 6.7$ & 0.172 \\
Agreeableness & $31.0 \pm 6.0$ & $30.0 \pm 5.2$ & $34.2 \pm 6.4$ & 0.067 \\
Conscientiousness & $32.7 \pm 6.1$ & $31.4 \pm 5.4$ & & \\
\hline
\end{tabular}

Data are presented as $n(\%)$ or mean \pm standard deviation.

Table 2. Odds ratios adjusted for sex and age to determine the factors associated with the cases

\begin{tabular}{lllllllr}
\hline Variables & OR & $95 \%$ CI & $p$ & H-L $(p)$ & AUC & $95 \%$ CI & $p$ \\
\hline Neuroticism & 1.19 & $1.08-1.32$ & 0.001 & 0.053 & 0.77 & $0.66-0.88$ & $<0.001$ \\
Extraversion & 0.94 & $0.87-1.02$ & 0.133 & 0.679 & 0.65 & $0.52-0.79$ & 0.015 \\
Openness to experience & 0.90 & $0.83-0.98$ & 0.015 & 0.571 & 0.69 & $0.56-0.82$ & 0.004 \\
Agreeableness & 0.95 & $0.87-1.04$ & 0.251 & 0.322 & 0.63 & $0.49-0.76$ & 0.037 \\
Conscientiousness & 0.91 & $0.83-1.00$ & 0.049 & 0.966 & 0.69 & $0.56-0.82$ & 0.004 \\
\hline
\end{tabular}

The H-L test indicates the calibration of the logistic regression model (whether the expected and observed probabilities of anal fissure are similar). This would be when $p>0.05$. On the other hand, the AUC is used to measure the discriminatory power of the model (range: $0.5-1$ ). Values around 0.7 are considered to be satisfactory. If we obtain a good calibration and discrimination, the results would correctly explain the association between anal fissure and personal traits. AUC, area under the ROC curve; CI, confidence interval; H-L, HosmerLemeshow test; OR, odds ratio.

and the mean age was 52.2 years. Regarding the characteristics of the NEO-FFI, the following mean scores were obtained: neuroticism, 17.1; extraversion, 27.3; openness to experience, 26.6; agreeableness, 31.0; conscientiousness, 32.7 .

Analysis of the differences between the cases and controls revealed a significantly higher mean neuroticism score in the cases $(p<0.001)$ and a higher openness to experience in the controls $(p=0.005)$. An increase in extraversion $(p=0.066)$ and conscientiousness $(p=0.067)$ in the controls was just short of reaching significance $(p<$ $0.05, p<0.10$; Table 1).

Adjustment of the results for sex and age (Table 2) resulted in 3 significant traits: (1) greater neuroticism in the cases (OR 1.19, $p<0.001$ ), (2) greater openness to experience in the controls (OR 0.90, $p=0.015$ ), and (3) greater conscientiousness in the controls (OR 0.91, $p=0.049$ ). The discrimination (AUC) of neuroticism was 0.77 and was the trait with the highest discrimination value. All the models used fit the data well (Hosmer-Lemeshow test did not reach significance in any of the cases).

\section{Discussion}

We found higher rates of neuroticism in patients with anal fissure than in the control group. The rates of openness to experience and conscientiousness, however, were higher in the control group. Neuroticism is also highlighted as the feature that best discriminates between patients with anal fissure and people who do not have this condition.

\section{Strengths and Limitations of the Study}

The main strength of this study is the clinical idea developed. Although other studies have used different approaches, assessing stress, psychopathology, and specific features [9-12], the clinical idea we developed could be a preliminary study to determine whether a high level of neuroticism is associated with a higher incidence of anal fissure, since finding a higher risk of fissure in people with higher levels of neuroticism leads us to consider carrying out a prospective study to assess this issue. Our study used a case-control design. Consequently, we were unable to 
assess this research question. We did use a complete questionnaire validated in Spanish with wide clinical application, which gives greater validity to our results. Nevertheless, other validated questionnaires could have been used $[13,14]$. In addition, the statistical power of the proposed comparison was very high, which implies high precision in the results. Finally, the magnitude of the effect and the discriminatory power of each of the traits were quantified.

With respect to limitations, as with all case-control studies, the control group must be chosen from the same population from which the cases are obtained. We were very careful, selecting patients who were undergoing surgery for another reason and hospital personnel, who could experience an anal fissure. In addition, the control group was homogeneous and very similar in age and sex to the case group. Furthermore, previous studies did not quantify the specific weight of traits in the risk of anal fissure $[9,10,12]$, which is important for making therapeutic decisions. In our study, this was addressed by determining the discrimination and calibration of the mathematical models used.

\section{Comparison with the Existing Literature}

When we assessed the relationship between neuroticism and anal fissure in the literature, we found some studies that approached this relationship with methodologies quite different from ours. First, a study carried out in Turkey observed that patients with anal fissure had a higher prevalence of type D personality [9]. Considering that the negative affectivity and social inhibition of type $\mathrm{D}$ components have been strongly associated with neuroticism [15], this is consistent with our findings. Another study, also conducted in Turkey [10], which did not work exclusively with patients with anal fissure but diverse anorectal disorders, obtained higher scores in harm avoidance and lower scores in self-directedness, scores that correlate with neuroticism [16].

In our study, the rates of openness to experience and conscientiousness were higher in the control group, which implies that these traits are less present in patients with anal fissure. In the literature, we found one study of a group of patients with different anorectal disorders that obtained higher scores in the control group for the dimension Persistence and Self-directedness, which correlate positively with conscientiousness [16]. However, in contrast to our results, scores for Self-transcendence, which correlate positively with openness to experience [16], were lower in the control group [10].

\section{Implications to Research and Clinical Practice}

Studies are needed to corroborate these results in order to better understand the differences in the personality traits of patients diagnosed with anal fissure planning to undergo medical and/or surgical treatment. Another line of research would be to analyze the effectiveness of the treatment according to personality traits, which could aid in developing improved action plans for this condition. In addition, as mentioned above, a prospective study is planned to assess the role of neuroticism in anal fissure. In this context, the etiopathogenesis of anal fissure is still not entirely clear. In humans, the internal anal sphincter is in a state of permanent partial contraction under the control of the autonomic nervous system, including the enteric nervous system. Several studies have shown that internal anal sphincter resting pressure is higher in patients with fissures compared to normal controls and that this hypertonicity is not secondary to local pain [17]. There is evidence that psychological stress leads to a substantial rise in anal canal pressure [18]. Similarly, the autonomic dysfunction induced by psychological factors such as hostility, anger, or anxiety can cause endothelial dysfunction $[19,20]$, in which the damaged endothelium produces vasoactive substances, such as endothelin-1, that contribute to an increase in basal tone and, consequently, ischemia of the posterior commissure $[21,22]$. In these cases, neuroticism has been associated with an increased risk of disease and systemic inflammation, with conscientiousness being the personality trait most consistently associated [23-25]. Inflammation is also a cause of endothelial dysfunction [26].

The psycho-neuroendocrine-immune approach is based on the concept of considering diseases from a holistic point of view: the different components (psyche, nervous system, endocrine system, and immune system) control the organs/systems, which in turn are influenced by a feedback mechanism, where all these physiological functions are subjected to the influence of psychological disorders in the "emotional motor system" [27, 28].

This study revealed that patients with anal fissure showed markedly neurotic personality traits with low conscientiousness. These findings, together with the knowledge that these traits correlate negatively with healthy behavior $[29,30]$ and treatment adherence [3133], enable the development and introduction of interventions to compensate for this negative relationship.

Knowing, for example, that a given patient has low conscientiousness, agreeableness, or openness to experience, or a predominance of neuroticism, may indicate a need for greater attention or motivation to increase the likelihood that that the person will adhere to the clinical aspects of the treatment program. A number of hygiene and dietary measures are common to the treatment of all types of anal fissures that are able to cure $>90 \%$ of acute fissures. These measures, however, must be continued beyond healing in acute fissures to avoid recurrence and chronicity. This continuation requires a second therapeutic step that involves the incorporation of long-term 
pharmacological measures that, together with the consistency in the application of conservative measures, facilitate the healing of the fissure without the need to proceed to the third therapeutic step, which is surgery. A multidisciplinary treatment team with specialized psychologists and nurses is needed to address the joint development of conscientiousness and health-related behaviors in order to potentially inoculate against the effects of low conscientiousness, facilitating a change in conscientiousness in favor of therapeutic effectiveness. Furthermore, the consideration of personality traits may enable improved therapeutic stratification. More studies are needed to assess whether patients with anal fissure who display these personality traits show greater dissatisfaction with medical interventions, are less compatible with medical therapy, or have inferior surgical outcomes.

\section{Conclusions}

Our findings indicate that patients with anal fissure present much higher levels of neuroticism (AUC >0.75) and lower levels of openness to experience and conscientiousness. Due to the lack of scientific evidence, more studies are needed to corroborate our results. If these results are confirmed, we should implement multidisciplinary treatment measures taking into account psychological assessments for greater therapeutic effectiveness.

\section{Acknowledgments}

The authors thank Maria Repice and Ian Johnstone for their help with the English version of the text.

\section{Statement of Ethics}

The study protocol was approved by the Ethics Committee of San Juan University Hospital in December 2016. All the participants gave their written informed consent.

\section{Disclosure Statement}

The authors have no conflicts of interest to declare.

\section{Funding Sources}

No external funding was received for this study.

\section{Author Contributions}

P.L.-P. designed the study and drafted the manuscript. A.C.-G. participated in the study design and helped draft the manuscript. A.P.-B. participated in the study design, critically reviewed the manuscript and performed the statistical analysis. F.N.-C., V.F.G.G., and A.F.C.-R. participated in the study design and critically reviewed the manuscript. All the authors read and approved the final version of the text to be submitted for publication.

\section{References}

1 Wald A, Bharucha AE, Cosman BC, Whitehead WE. ACG clinical guideline: management of benign anorectal disorders. Am J Gastroenterol. 2014 Aug;109(8):1141-57.

2 Utzig MJ, Kroesen AJ, Buhr HJ. Concepts in pathogenesis and treatment of chronic anal fissure - a review of the literature. Am J Gastroenterol. 2003 May;98(5):968-74.

3 Stewart DB Sr, Gaertner W, Glasgow S, Migaly J, Feingold D, Steele SR. Clinical Practice Guideline for the Management of Anal Fissures. Dis Colon Rectum. 2017 Jan;60(1):714.

4 Arroyo A, Montes E, Calderón T, Blesa I, Elía M, Salgado G, et al. Treatment algorithm for anal fissure. Consensus document of the Spanish Association of Coloproctology and the Coloproctology Division of the Spanish Association of Surgeons. Cir Esp. 2018 May; 96(5):260-7.

5 McCrae RR, Costa PT Jr, Ostendorf F, Angleitner A, Hrebícková M, Avia MD, et al. Nature over nurture: temperament, personality, and life span development. J Pers Soc Psychol. 2000 Jan;78(1):173-86.

6 Costa PT, McCrae RR. NEO-PI-R. Professional Manual. Revised NEO personality Inventory and NEO Five Factor Inventory. Odessa, Ukraine: Psychological Assessment Resources; 1992.
7 McCrae RR, Costa PT. The five-factor theory of Personality. In: John OP, Robins RW, Pervin LA, editors. Handbook of personality. New York, NY, USA. Guilford; 2008.

8 Costa PT Jr, McCrae RR. Inventario de Personalidad NEO Revisado (NEO PI-R). Inventario NEO reducido de Cinco Factores (NEOFFI). Madrid, Spain: Manual TEA; 1999.

9 Sit M, Yilmaz EE, Canan F, Yildirim O, Cetin MM. Health-related quality of life in patients with anal fissure: effect of type D personality. Prz Gastroenterol. 2014;9(2):93-8.

10 Akçay B, Gül V, Özer S. Temperament and character traits in patients with anorectal disorder. J Surg Med. 2018;2(1):17-22.

11 Cioli VM, Gagliardi G, Pescatori M. Psychological stress in patients with anal fistula. Int J Colorectal Dis. 2015 Aug;30(8):1123-9.

12 Arısoy Ö, Şengül N, Çakir A. Stress and psychopathology and its impact on quality of life in chronic anal fissure (CAF) patients. Int J Colorectal Dis. 2017 Jun;32(6):921-4.

13 Cloninger CR, Przybeck TR, Svrakic DM, Wetzel RD. The Temperament and Character Inventory (TCI): a guide to its development and use. St. Louis, Missouri, USA: Center for Psychobiology of Personality; 1994.

14 Eysenck HJ, Eysenck SB. Manual of the Eysenck Personality Questionnaire. Los Ange- les, CA, USA: EdITS/Educational and Industrial Testing Service; 1994.

15 De Fruyt F, Denollet JK. Personality: a fivefactor model perspective. Psychol Health. 2002;17(5):671-83.

16 De Fruyt F, De Wiele LV, Van Heeringen C. Cloninger's psychobiological model of temperament and character and the Five-Factor Model of personality. Pers Individ Dif. 2000; 29(3):441-52.

17 Shafik A. A new concept of the anatomy of the anal sphincter mechanism and the physiology of defecation. XV. Chronic anal fissure: a new theory of pathogenesis. Am J Surg. 1982 Aug; 144(2):262-8.

18 Spadari RC, Cavadas C, de Carvalho AE, Ortolani D, de Moura AL, Vassalo PF. Role of Beta-adrenergic Receptors and Sirtuin Signaling in the Heart During Aging, Heart Failure, and Adaptation to Stress. Cell Mol Neurobiol. 2018 Jan;38(1):109-20.

19 Lin TK, Weng CY, Wang WC, Chen CC, Lin IM, Lin CL. Hostility trait and vascular dilatory functions in healthy Taiwanese. J Behav Med. 2008 Dec;31(6):517-24.

20 Narita K, Murata T, Hamada T, Takahashi T, Omori M, Suganuma N, et al. Interactions among higher trait anxiety, sympathetic activity, and endothelial function in the elderly. J Psychiatr Res. 2007 Aug;41(5):418-27. 
21 Maria G, Brisinda D, Ruggieri MP, Civello IM, Brisinda G. Identification of anti-endothelial cell antibodies in patients with chronic anal fissure. Surgery. 1999 Sep;126(3):53540.

22 Lim CH, Shin HK, Kang WH, Jeong SK, Yang HK. Is There Any Relationship between the Chronicity of Chronic Anal Fissure and Endothelin-1? J Korean Soc Coloproctol. 2011 Oct;27(5):237-40.

23 Schaller M. The behavioural immune system and the psychology of human sociality. Philos Trans R Soc Lond B Biol Sci. 2011 Dec: 366(1583):3418-26.

24 Sutin AR, Terracciano A, Deiana B, Naitza S, Ferrucci L, Uda M, et al. High neuroticism and low conscientiousness are associated with interleukin-6. Psychol Med. 2010 Sep;40(9) 1485-93.
25 Luchetti M, Barkley JM, Stephan Y, Terracciano A, Sutin AR. Five-factor model personality traits and inflammatory markers: new data and a meta-analysis. Psychoneuroendocrinology. 2014 Dec;50:181-93.

26 Steyers CM 3rd, Miller FJ Jr. Endothelial dysfunction in chronic inflammatory diseases. Int J Mol Sci. 2014 Jun;15(7):11324-49.

27 Straub RH, Cutolo M. Psychoneuroimmunology-developments in stress research. Wien Med Wochenschr. 2018 Mar;168(3-4): 76-84.

28 Pescatori M, Podzemny V, Pescatori LC, Dore MP, Bassotti G. The PNEI holistic approach in coloproctology. Tech Coloproctol. 2015 May;19(5):269-73

29 Papachristou H, Nederkoorn C, Jansen A. Neuroticism and Negative Urgency in Problematic Alcohol Use: A Pilot Study. Subst Use Misuse. 2016 Sep;51(11):1529-33.
30 Allen MS, Walter EE. Linking big five personality traits to sexuality and sexual health: A meta-analytic review. Psychol Bull. 2018 Oct; 144(10):1081-110.

31 Axelsson M, Brink E, Lundgren J, Lötvall J. The influence of personality traits on reported adherence to medication in individuals with chronic disease: an epidemiological study in West Sweden. PLoS One. 2011 Mar 6(3):e18241.

32 Emilsson M, Berndtsson I, Lötvall J, Millqvist E, Lundgren J, Johansson A, et al. The influence of personality traits and beliefs about medicines on adherence to asthma treatment. Prim Care Respir J. 2011 Jun;20(2):141-7.

33 O'Cleirigh C, Ironson G, Weiss A, Costa PT Jr. Conscientiousness predicts disease progression (CD4 number and viral load) in people living with HIV. Health Psychol. 2007 Jul; 26(4):473-80. 